\title{
The effect of helicopter low altitude cruise on the efficiency of radar detection
}

\author{
Jun-chao Yuan ${ }^{1,}$ a, *, Xiao-kuan Zhang ${ }^{1}$, Shuai Wang ${ }^{2}$ \\ ${ }^{1}$ School of Air Force Engineering University, Xi'an 710051, China; \\ ${ }^{2}$ Unit 94245 of PLA, Shangdong,Rizhao 276809, China; \\ a17791252698@163.com, \\ *corresponding author
}

Keywords: attack helicopter, RCS sequence, low altitude cruise, ground clutter interference

\begin{abstract}
According to the flight characteristics of the attack helicopter cruising section, the cruise tracks at three different heights are imitated. Then, the variation of the RCS sequence of helicopter with time is simulated. The strong ground clutter is taken into account when helicopters are cruising at low altitude. The signal strength of ground clutter is estimated. Finally, the radar detection probability of helicopter penetration at three cruise heights is calculated. The results show that when the attack helicopter flies at low altitude, ground clutter intensity is largely restricted to the detection performance of radar. The lower the cruise height is, the worse the radar detection efficiency is, and it will become more conducive to improving the battlefield survivability of helicopters.
\end{abstract}

\section{Introduction}

Attack helicopters are now a major component of the air power of all countries. It plays a more and more important role in modern combat because of the characteristics, low, slow and small. Low means the flying altitude is low. Slow means the cruising speed is slow. Small is that the Radar cross section (RCS) is small. Therefore, when the radar detects helicopters, there is limitation of sight distance. It is difficult to detect weak signal, and there is multipath interference effect [1]. In the paper, the influence of helicopter cruise on efficiency of radar detection under different altitude is studied by simulation of ground clutter and RCS sequence of helicopter.

\section{The main features of an attack helicopter}

An attack helicopter is an aircraft loaded with many weapons. It has the characteristics of wide attack range, multiple weapon, good usability, etc. when attack helicopter has cruising flight at low attitude, the radar detection range is limited by the sight distance due to the influence of the curvature of the earth. As the result, early warning radar usually raises the erection height. Furthermore, when the main beam of radar detection is on the ground, the echo contains a lot of ground clutter which makes it more difficult to detect. The radar cross section of attack helicopters is small. The typical helicopter target is among $\mathrm{L}$ and S band but the RCS is the direction of nasal cone is only $0.1 \mathrm{~m}^{2} \sim 0.5 \mathrm{~m}^{2}$.

\section{RCS simulation of attack helicopter}

Usually, the target RCS is considered as an average constant in simulation, but the actual target is not a uniform scatterer. The RCS is not only related to the working wavelength of radar and polarization, but also to the attitude angle of target and the line of sight of radar. In the more accurate simulation, it is necessary to consider the RCS time series of the target changing with the radar line of sight angle. The paper assumes that the ground early warning radar is vertical polarization of $\mathrm{P}$ band. Then, taking an attack helicopter as an example and using FEKO electromagnetic simulation 
software, MOM (method of moment) is used to calculate the electromagnetic scattering characteristics of the helicopter in P band. At last, target database of static RCS for the whole space is obtained. The color map of RCS distribution of the whole airspace target is shown in figure 1. According to the diagram, the head of the helicopter has small RCS, however the lateral RCS is large.

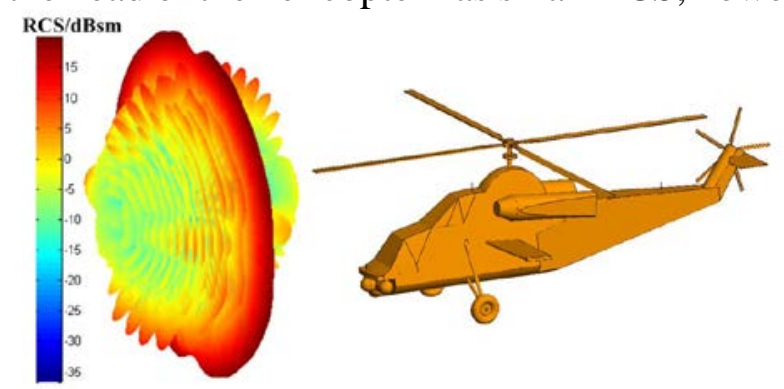

Fig. 1 Full space static RCS distribution of helicopter in P band

Firstly, it is assumed that the helicopter cruise section makes uniform straight line flight; the initial position is $30 \mathrm{~km}$ from the radar, and the flying speed is $260 \mathrm{~km} / \mathrm{h}$. Three different cruising altitude $(50 \mathrm{~m}, 100 \mathrm{~m}, 150 \mathrm{~m})$ get three tracks and real-time changes of the radar line of sight angle of each track. Finally, the dynamic RCS sequence of the helicopter is obtained by the static RCS database of the whole target space (figure 2). The altitude of the long-range early warning radar is set at $500 \mathrm{~m}$ during simulation. As shown is figure 2, the higher the cruising altitude is, the smaller the RCS is. This is because the higher the cruise height, the closer the radar beam to the strong stealth area of the helicopter's head where the RCS is small according to figure 1.

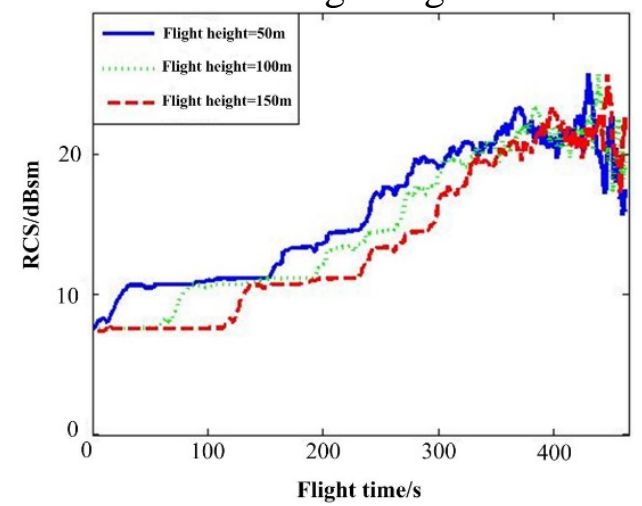

Fig. 2 RCS sequence of helicopter at different cruising altitude

\section{Clutter simulation}

The ground clutter received by radar is mainly formed by scattering of electromagnetic waves from the earth's surface. When discussing the influence of clutter on radar, clutter backscattering coefficient $\sigma_{0}$ is usually used to describe quantitatively. ${ }^{\sigma_{0}}$ represents the average radar cross section of a unit surface area, which is related to topography, incidence angle, wave band of radar etc[3]. The paper uses the backscattering coefficient model of ground clutter proposed by Morchin. The model considers both the effect of different topography on backscattering coefficients and also the specular component of mirror reflection when the incidence is almost vertical. What is more, the model is more accurate compared with the classical ${ }^{\sigma_{0}}$ model which has the constant $\gamma$. The expression is as follows.

$$
\sigma_{0}=\frac{A \sigma_{\mathrm{c}}^{0} \sin \theta}{\lambda}+u \cot ^{2} \beta_{0} \exp \left[-\frac{\tan ^{2}(B-\theta)}{\tan ^{2} \beta_{0}}\right]
$$

In the above equation $u=f^{1 / 2} / 4.7 . f$ is operating frequency of radar with unit GHz. $\lambda$ is operating wavelength of radar. $\theta$ is grazing angle. $A 、 B 、 \beta_{0} 、 \sigma_{\mathrm{c}}^{0}$ are model parameters related to topography. The values are $0.004 、 \pi / 2 、 0.2 、 1$. 
Clutter intensity of ground ${ }^{\sigma_{\mathrm{c}}}$ (also known as clutter RCS) is[4]

$$
\sigma_{\mathrm{c}}=A_{\mathrm{s}} \cdot \sigma_{0}
$$

The area of resolution element of radar

$$
A_{\mathrm{s}}=\frac{c \tau}{2} \cdot \frac{1}{\cos \theta} \cdot R \cdot \theta_{\beta 0.5}
$$

$C$ is propagation velocity of electromagnetic wave. $\tau$ is the width of radar transmit pulse; $\theta_{\beta 0.5}$ is the half-power width of radar beam pitch. $R$ is the distance between radar and the target.

Figure 3 shows the real time variation of intensity $\sigma_{c}$ of the ground clutter with helicopter cruise. As shown in the diagram, the intensity of ground clutter increases rapidly as the distance between the helicopter and radar decreases. It is mainly because that the grazing angle of radar beam increases. The higher the cruise height of helicopters, the weaker the clutter, which is in line with the actual situation.

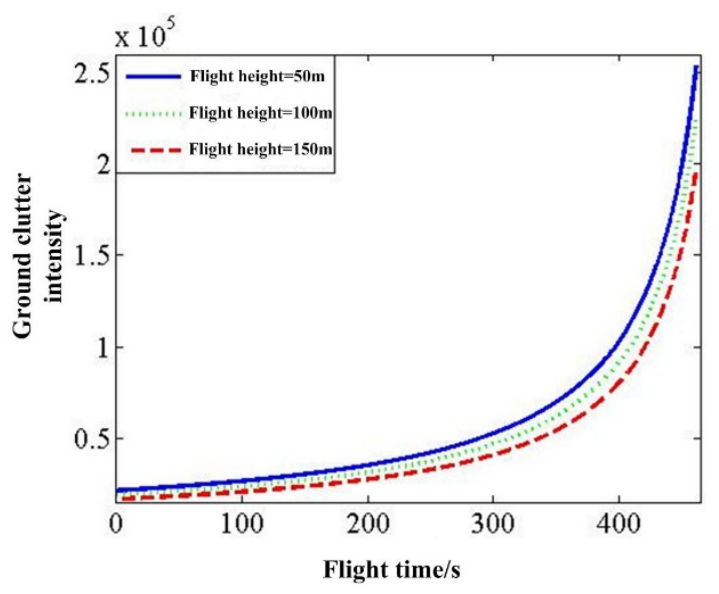

Fig. 3 Variation of ground clutter intensity during helicopter Cruise

\section{Model of radar detection}

\subsection{Module of integrated signal-to-noise ratio.}

Without considering the multipath effect, the radar receiver's processing signal mainly has the target-echo signal, the ground clutter signal and the receiver noise[4,5].

(1) signal power of the target echo $P_{\mathrm{r}}$

$$
P_{\mathrm{r}}=\frac{P_{\mathrm{t}} G^{2} \lambda^{2} \sigma}{(4 \pi)^{3} R^{4} L}
$$

$P_{\mathrm{t}}$ is transmit power of radar. $G$ is antenna gain. $\lambda$ is operating wavelength of radar. $\sigma$ is target RCS. $L$ is loss of radar system.

(2) signal power of ground clutter $P_{c}$

$$
P_{\mathrm{c}}=\frac{P_{\mathrm{t}} G^{2} \lambda^{2} \sigma_{\mathrm{c}}}{(4 \pi)^{3} R^{4} L}
$$

(3) signal power of receiver noise $P_{\mathrm{n}}$

$$
P_{\mathrm{n}}=k F_{\mathrm{n}} T_{0} B_{\mathrm{n}}
$$

In the above equation, $k$ is Boltzmann constant. $F_{\text {n }}$ is receiver noise factor. $T_{0}$ is temperature of system noise. ${ }^{B_{\mathrm{n}}}$ is equivalent noise bandwidth of the receiver.

Integrated signal to noise ratio is as follows. $I$ is improvement factor of the clutter.

$$
S / N=P_{\mathrm{r}} /\left(P_{\mathrm{c}} / I+P_{\mathrm{n}}\right)
$$




\subsection{Calculation module of detection probability.}

The helicopter uses a Swerling IV relief model [6]. The detection probability PD can be obtained by the following equation [7].

$$
\begin{gathered}
P_{\mathrm{D}}=1-\left[\gamma_{0}+\left(\frac{S / N}{2}\right) n_{\mathrm{p}} \gamma_{1}+\left(\frac{S / N}{2}\right)^{2} \frac{n_{\mathrm{p}}\left(n_{\mathrm{p}}-1\right)}{2 !} .\right. \\
\left.\gamma_{2}+\ldots+\left(\frac{S / N}{2}\right)^{n_{\mathrm{p}}} \gamma_{n_{\mathrm{p}}}\right]\left(1+\frac{S / N}{2}\right)^{-n_{\mathrm{p}}} \\
\gamma_{i}=\Gamma_{\mathrm{I}}\left(\frac{V_{\mathrm{T}}}{1+(S / N) / 2}, n_{\mathrm{p}}+i\right)
\end{gathered}
$$

In above equations, ${ }^{\Gamma_{\mathrm{I}}}$ stands for incomplete function. np is accumulated Number of pulses. $V_{\mathrm{T}}$ is detection threshold which can be got by a recursive formula in the Newton-Raphson method.

By using the recursion formula

$$
\Gamma_{\mathrm{I}}(x, i+1)=\Gamma_{\mathrm{I}}(x, i)-\frac{x^{\mathrm{i}}}{i ! \exp (x)}
$$

Only $\gamma_{0}$ needs to use equation (9). Except ${ }^{\gamma_{0}}, \gamma_{\mathrm{i}}$ can be calculated by the following equations

$$
\begin{aligned}
& \gamma_{\mathrm{i}}=\gamma_{\mathrm{i}-1}-A_{1} \quad i>0 \\
& A_{1}=\frac{V_{\mathrm{T}} /(1+((S / N) / 2)}{n_{\mathrm{p}}+i-1} A_{\mathrm{l}-1} \quad i>1 \\
& A_{1}=\frac{\left(V_{\mathrm{T}} /(1+((S / N) / 2))^{n_{\mathrm{p}}}\right.}{n_{\mathrm{p}} ! \exp \left(V_{\mathrm{T}} /(1+((S / N) / 2))\right.} \\
& \gamma_{0}=\Gamma_{\mathrm{I}}\left(\frac{V_{\mathrm{T}}}{1+(S / N) / 2}, n_{\mathrm{p}}\right)
\end{aligned}
$$

\subsection{Simulation results of radar detection probability.}

The parameters of remote warning simulation radar are set as: Frequency $f=435 \mathrm{MHz}$, transmitting power $P_{\mathrm{t}}=600 \mathrm{~kW}$, antenna gain $G=40 \mathrm{~dB}$, noise factor of receiver $F_{\mathrm{n}}=5 \mathrm{~dB}$, temperature of system noise $T_{0}=496$, improvement factor of the clutter $I=58 \mathrm{~dB}$, transmitted pulse width $\tau=50 \mu \mathrm{s}$ 。Loss of system is ignored. It needs to be explained that the target is $120 \mathrm{~km}$ far away from radar which can meet the sight condition of radar. When the accumulated number of pulse is 5, and the false-alarm probability $P_{\mathrm{fa}}=10^{-6}$, The simulation results of radar detection probability are shown in Figure 4.

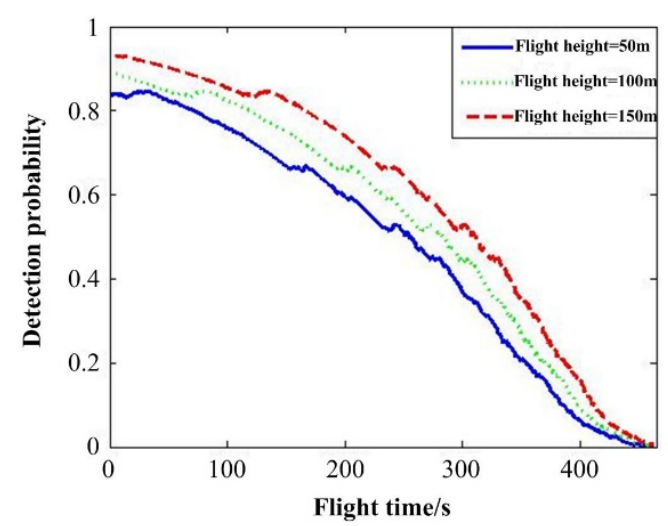

Fig. 4 detection probability of radar

According to figure 4, when the same helicopter reach the radar horizontal projection distance, cruise height is higher, the detection probability of radar is bigger. Figure 2 shows that the higher the 
cruise height is, the smaller the target RCS sequence is, and the smaller the signal power of target echo is. However, the higher the cruise height is, the weaker the ground clutter is, and the larger the radar detection probability is. With the decrease of the distance between the helicopter and radar, the detection probability of radar becomes very small. It is because that when the distance is small, the clutter is strong and the target echo is submerged in noise. Therefore, compared to target RCS, clutter intensity is the main factor that affects radar detection of small targets at low altitude. In all, improving the clutter improvement factor, reducing the clutter intensity and improving signal to clutter power ratio is the key to improve the detection of low altitude targets in clutter.

\section{Summary}

The paper takes into account of the influence of ground clutter and target scattering characteristics on radar detection efficiency at low altitude penetration. By simulating the change of radar detection probability at different cruising altitude of helicopter, it can be obtained that the lower the cruise height is, the more difficult the radar detection is and the better it is for penetration. The conclusion is in line with the actual situation. The simulation results show that ground clutter has great influence on radar detection. It is also one of reasons why suppressing strong clutter and detecting small targets under strong clutter are hotpots in the study field of radar.

\section{References}

[1] Wang L J, Guo J M, Gao C M, et al. Research on problems of conventional ground based radar detecting helicopter[J], Aerospace Electronic Warfare, 2009, 25(6): 8-10.

[2] Fu A Q, Li J X, Gao Z F, et al. Clutter's simulation and signal reconstruction based on backward scattering factor model[J], Aerospace Electronic Warfare, 2010, 26(5): 26-30.

[3] Pan L, Fu Q, Zhang J. Simulation and calculation on a kind of ground-clutter spectrum[J], Systems Engineering and Electronics, 2005, 27(4): 586-590.

[4] Fan Y Z, Zhang W H, Xie D C. Simulation Analysis of Cruise Missile Flight Condition's Effects on Tracking Irradiation Radar Detecting Performance[J], Journal of System Simulation, 2008, 20(15): 3927-3930.

[5] Yuan H, Tao J F, An L. Research of Radar Detection Model under Complex Battle Environments[J], Computer Measurement \& Control, 2012, 20(11): 2999-3004.

[6] Dai H S, Ye B F, Li X, et al. An Evaluate Way of Radar Capability to Detect helicopter[J]. Journal of CAEI, 2008, 3(4): 403-406.

[7] Bassem R. Mahafza, Atef Z. Elsherbeni. MATLAB simulation based on design of radar system[M]. Beijing: Electronic Industry Press, 2009. 